4. L. Tingting, H. Jianjun, and H. Sailing, A novel triplexer design based on arrayed waveguide grating. Chin J Semicond 27 (2006), 368.

5. L. Wenhua1, L. Guangbo, J. Kemiao, et al. Design and fabrication of $1 \times 32$ silica-based arrayed waveguide grating. Chin J Semicond 26 (2005), 1798.

6. M.R. Amersfoort, C.R. de Boer, F.P.G. van Ham, et al. Phased-array wavelength demultiplexer with flattened wavelength response. Electron Lett 30 (1994), 300.

7. M.R. Amersfoort, J.B.D. Soole, H.P. LeBlance, et al. Passband broadening of integrated arrayed waveguide filters using multimode interference couplers. Electron Lett 32 (1996), 449.

8. Y.P. Ho, H. Li, and Y. Chen. Flat channel-passband-wavelength multiplexing and demultiplexing devices by multiple-Rowland-circle design. IEEE Photon Technol Lett 9 (1997), 342.

9. T. Kamalakis and T. Sphicopoulos. An efficient technique for the design of an arrayed-waveguide grating with flat spectral response. J Lightwave Technol 19 (2001), 1716.

10. C.S. Ma, H.M. Zhang, D.M. Zhang, et al. Box-like spectral response of arrayed waveguide grating multiplexers. Opt Commun 249 (200), 209.

11. W.B. Guo, C.S. Ma, D.M. Zhang, et al. Parameter optimization and structural design of polymer arrayed waveguide grating multiplexer. Opt Commun 201 (2002), 45.

12. C.S. Ma, X.Y. Wang, H.M. Zhang, et al. An efficient technique for analyzing transmission characteristics of arrayed waveguide grating multiplexers. Opt Quantum Electron 36 (2004), 759.

(C) 2010 Wiley Periodicals, Inc.

\section{SPHERICAL LENS ANTENNA DESIGNS WITH PARTICLE SWARM OPTIMIZATION}

\author{
Benjamin Fuchs, Ruzica Golubovic, Anja K. Skrivervik, \\ and Juan R. Mosig \\ Laboratory of Electromagnetics and Acoustics (LEMA), Ecole \\ Polytechnique Fédérale de Lausanne (EPFL), Bâtiment ELB, \\ Station 11, CH-1015 Lausanne, Switzerland; Corresponding \\ author: benjamin.fuchs@univ-rennes1.fr
}

Received 7 October 2009

ABSTRACT: A design procedure for spherical lens antennas is described. A particle swarm optimization (PSO) algorithm is coupled to a mode matching technique based on spherical wave expansion to analyze the lens antennas. The proposed methodology is applied to three optimization problems using real-number and binary PSO. First, the maximization of the directivity of Luneburg lens antennas is addressed. Then, amplitude shaped radiation patterns are synthesized by optimizing both amplitude and position of each element of an array that illuminates a lens. Finally, a dual-beam reconfigurable lens antenna is optimized. By only switching properly the elements of an array, the lens antenna radiates either a directive or a sectoral beam. Numerical comparisons with a full wave commercial software successfully validate the proposed design procedure. (C) 2010 Wiley Periodicals, Inc. Microwave Opt Technol Lett 52: 1655-1659, 2010; Published online in Wiley InterScience (www.interscience.wiley.com). DOI 10.1002/mop.25278

Key words: lens antennas; optimization; inhomogeneous lenses; millimeter wave antenna

\section{INTRODUCTION}

Lens antennas are widely used in millimeter wave applications such as automotive radar [1, 2], satellite transmissions [3], or indoor communications [4].

Lens antennas typically consist of a dielectric lens (spherical or hemispherical) fed by a primary source (single feed or array). Dielectric lenses are conventionally used as focusing devices to enhance the directivity of the primary source. They can also be used to generate multiple beams from a feed array. More recently, shaped beams have been obtained with spherical lens antennas [5-7] opening a new range of applications.

In this article, a design procedure for the optimization of spherical lens antennas is described and applied to obtain directive, shaped, and reconfigurable beams. The proposed procedure uses for analysis a fully analytical method (the mode matching technique-MMT-based on spherical wave functions), and then combines it with a particle swarm optimization (PSO) algorithm. Because the MMT kernel is fast and accurate to analyze spherical lens antennas and the PSO algorithm is very efficient for solving optimization problems with a medium-sized number of unknowns, this combination reveals to be very well-suited for such design problems.

This article is organized as follows. First, the design procedure, namely the analysis technique and the optimization methods, is described in Section 2. Then three lens antenna optimization problems are thoroughly solved in Section 3. The first concerns the directivity maximization of Luneburg lens antennas and the second the synthesis of amplitude shaped radiation patterns with a lens fed by an array. Finally, the third and the last deals with the optimization of a dual-beam reconfigurable lens antenna. Conclusions are drawn in Section 4.

\section{ANALYTICAL FORMULATION}

The flowchart describing the design procedure is represented in Figure 1. The lens antenna analysis is done using a MMT kernel and constitutes the inner step in an optimization loop driven by the PSO algorithm. To link the optimization algorithm to the physical problem, a cost function has to be defined. The cost function measures in a single number the optimality of the solution.

The main input parameters of this procedure are the lens antenna configuration, namely the primary feeds (type, number, and position), the lens itself (geometry and material parameters), and the working frequency. In addition, the PSO algorithm needs some internal settings to be specified (variables to optimize and their respective ranges). As usual, the stopping criterion is either a cost function threshold or a maximum number of iterations.

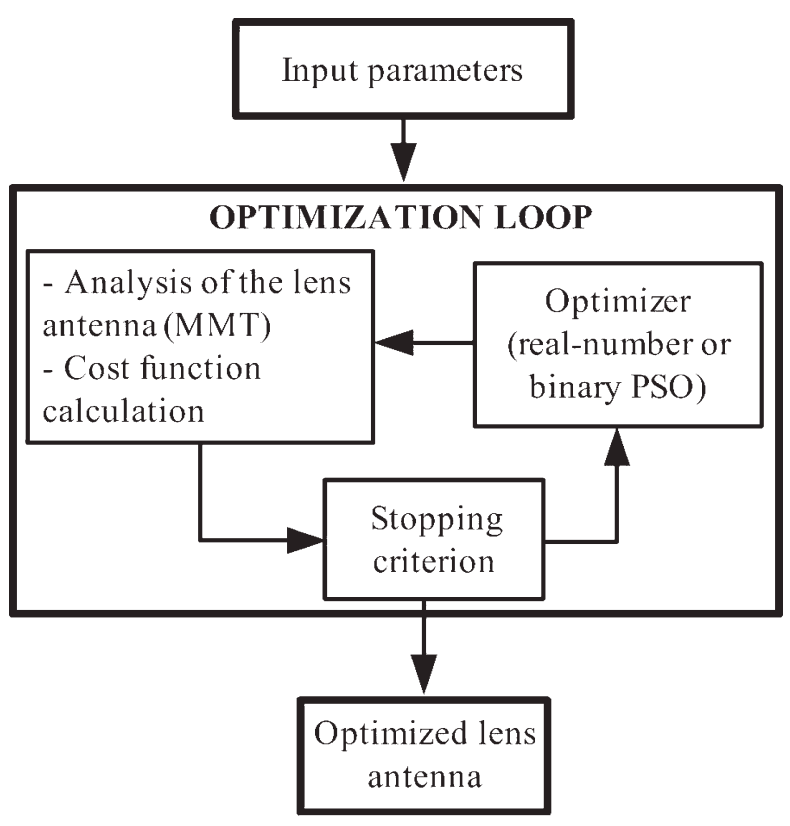

Figure 1 Flowchart describing the design procedure of spherical lens antennas 


\subsection{Analysis Technique}

A MMT based on spherical wave functions is used to analyze spherical lens antennas and this method has already been applied and validated (both numerically and experimentally) to compute the scattering by spherically stratified lenses fed by general source configurations [7-10]. The major interest of this method is that the analytical computation of the far-field radiation patterns and directivity can include realistic primary sources within a reasonable computation load. Two source models are used in this article. First, a complex source point model. This is an exact solution of the Helmholtz equation, which allows to simulate the directive nature of a realistic feed in a very simple way, because increasing the imaginary part of the source coordinate directly and narrows the radiated beam [11]. As a second source, a WR10 open-ended waveguide, whose electromagnetic field is known analytically on its aperture, is used to simulate a realistic feeding device.

\subsection{Optimization Method (PSO)}

The PSO is a relatively new global optimization method, developed by Kennedy and Eberhart [12], which has recently been successfully applied to electromagnetic problems ([13, 14]).

The PSO algorithm is based on the analogy with the social behavior of the bees' swarms. Each bee is an individual called particle and all the particles that are searching in the optimization space form the swarm. Each particle updates its position $x$ depending on its previous position, on the best position found by itself $p_{\text {best }}$ and on the best position found by the whole swarm $g_{\text {best }}$. Here follows a succint description of the specific PSO version developed for this article.

Assume that at the given iteration $t$ a particle is located at $x_{t}$. The particle knows its previous position $x_{t-1}$, and the current best position achieved by itself, $p_{\text {best }}$ and by the swarm, $g_{\text {best }}$. It has therefore three logical directions to progress:

a. follows its own inertia, defined by the difference $x_{t}-$ $x_{t-1}$,

b. approaches its best result by following the difference $p_{\text {best }}$ $-x_{t}$, and

c. approaches the swarm best result by following the difference $g_{\text {best }}-x_{t}$.

In practice, a good compromise is to make the particle progress along a linear combination of these three possibilities (Fig. 2). Hence, the particle position is updated as:

$$
x_{t+1}=x_{t}+v_{t+1},
$$

with

$$
\begin{aligned}
v_{t+1}=c_{0} \cdot\left(x_{t}-x_{t-1}\right)+c_{1} \cdot \operatorname{rand}() & \cdot\left(p_{\text {best }}-x_{t}\right) \\
& +c_{2} \cdot \operatorname{rand}() \cdot\left(g_{\text {best }}-x_{t}\right) .
\end{aligned}
$$

Quite logically the coefficients or weights $c_{0}, c_{1}$, and $c_{2}$ are called inertial, cognitive, and social coefficients, respectively. The absolute values of the coefficients define the acceleration characteristics. For instance, the choice $c_{0}=1, c_{1}=c_{2}=0$ corresponds to a uniform particle motion, blindly dominated by inertia. A more critical factor is the consideration of the coefficient ratios and of their evolution during the optimization process. For instance, it has been demonstrated (see [13,14] for full details about the PSO algorithm and its various schemes) that, frequently better performances are obtained when the value of the inertial coefficient $c_{0}$ (frequently called $w$ in the literature) decreases during the iterative procedure. Also, as in most societies, a good start

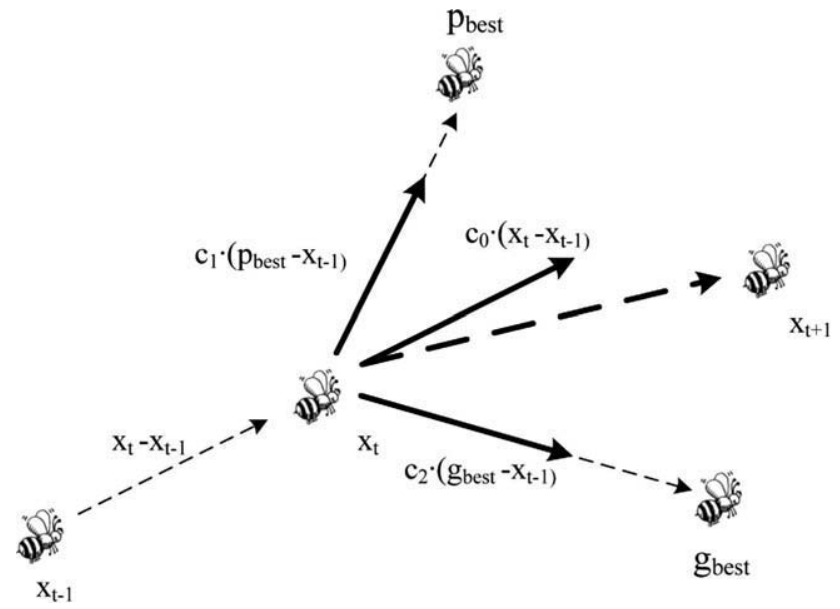

Figure 2 Updating the particle's position

is provided by selecting identical values for $c_{1}$ and $c_{2}$. But a degree of randomness between selfish and social behavior is most welcome (as in many societies) and can be achieved by multiplying $c_{1}$ and $c_{2}$ by rand(), a function that generates uniformly distributed random numbers between 0 and 1 . In that way, some particles will show somewhat unexpected deviating from the global trend and exploring some a priori unpromising sections of the optimization space. Finally, the total velocity may undergo a normalization process to fit the optimization space.

A worth mentioning point is that in the most commonly encountered situations, $x_{t}, p_{\text {best }}$, and $g_{\text {best }}$ are $1 \mathrm{D}$-arrays of realvalued elements, the dimension of the vectors corresponding to the dimension of the continuous optimization space. But more recently, PSO has been also successfully applied to discrete problems involving binary variables and parameters. In this article, both real-number and binary PSO are used. The implemented PSO parameters are now detailed for each case.

2.2.1. Real-Number Version. A time-varying $c_{0}$ decreasing from 0.9 to 0.4 over the course run is set, as suggested in [13]. $c_{1}$ and $c_{2}$ are equal to 1.5 . The maximal particle's velocity is set to be equal to the dynamic range for each dimension of the optimization space. A boundary condition, known as invisible wall, is applied for particles that go out of the solution space [13]. Because $c_{1}$ and $c_{2}$ are multiplied by a random number $\operatorname{rand}()$, their mean value $(0.75)$ roughly corresponds to the value taken by $c_{0}$ in the intermediate steps of optimization process.

2.2.2. Binary Version. For some optimization problems, realnumber PSO cannot be used. For instance, binary PSO is required to optimize an array whose elements take only $\mathrm{ON}$ or OFF states. In binary PSO, a particle moves in a solution space that is restricted to 0 and 1 for each dimension. Equation (2) is used with the distinction that now $x, p_{\text {best }}$, and $g_{\text {best }}$ are binaryvalued vectors. $c_{1}$ and $c_{2}$ remain equal to 1.5 , whereas $c_{0}$ is set to 1 because in this case a time-varying $c_{0}$ does not improve the convergence, as shown in [13]. As the particles' velocities $v$ are still real-valued vectors, the particles' positions can no more be updated according to Eq. (1). For that purpose, Kennedy and Eberhart in [15] suggested to use an intermediate variable.

$$
S\left(v_{m n, t}\right)=\frac{1}{1+\exp \left(-v_{m n, t}\right)},
$$

where $v_{m n, t}$ is the $n$th bit velocity of the $m$ th particle in the $t$ th iteration. The particle's position is then computed as follows: 


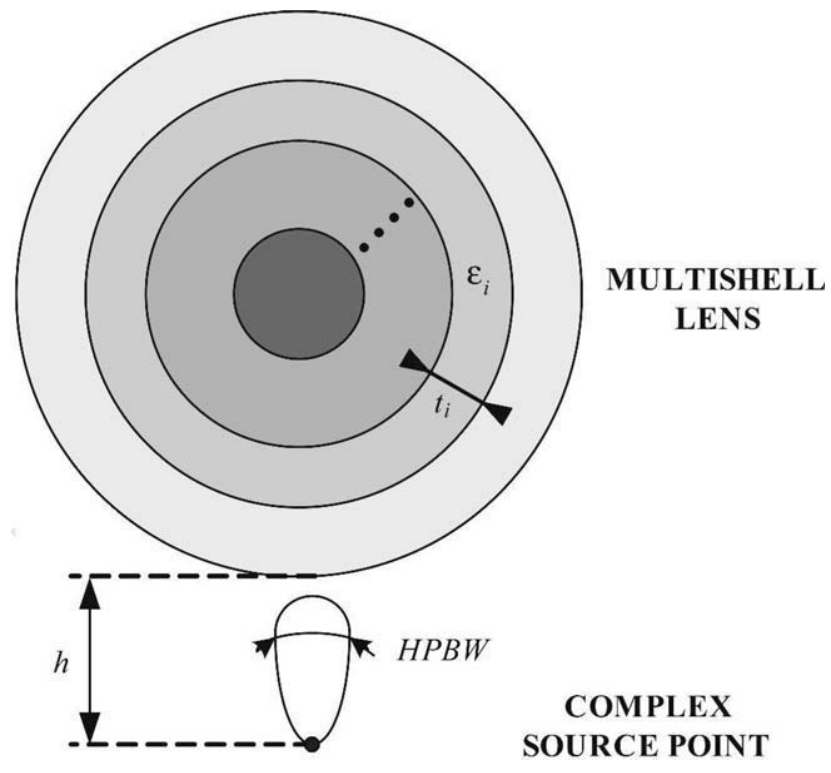

Figure 3 Geometry of the Luneburg lens optimization problem. A multishell lens is fed by a complex source point beam. $\varepsilon_{\mathrm{i}}$ and $t_{\mathrm{i}}$ are respectively the permittivity and thickness of the ith shell of the lens. HPBW stands for the half power beam width of the feed and $h$ is the distance between the feed and the lens

$$
\text { if }\left(\operatorname{rand}()<S\left(v_{m n, t}\right)\right) \text {, then } x_{m n, t}=1 \text { else } x_{m n, t}=0 \text {. }
$$

Contrary to real-number PSO, a high maximum velocity reduces the range explored by a particle in binary PSO. This value is set to 6.0 , as recommended in [15].

\section{APPLICATION TO LENS ANTENNA OPTIMIZATION PROBLEMS}

In this section, the PSO coupled to the MMT is applied to optimize the design of three lens antenna configurations.

\subsection{Directivity Maximization of Luneburg Lenses}

Luneburg lenses have a continuous permittivity variation [16] that is practically approximated by a finite number of concentric homogeneous shells. The usual problem is how to choose each shell parameter (thickness $t_{\mathrm{i}}$ and permittivity $\varepsilon_{\mathrm{i}}$ ) to maximize the lens antenna directivity.

This optimization problem has already been addressed by several researchers [17-19]. This article provides by the first time

TABLE 1 Input and Optimized Parameters of Luneburg Lens Optimization Problem

\begin{tabular}{lc}
\hline Fixed Parameter & Value \\
\hline Lens diameter $\left[\lambda_{0}\right]$ & $D=30$ \\
Number of shells & $N=5$ \\
\hline Parameter to optimize & Boundaries \\
\hline Normalized thicknesses, $t_{\mathrm{i}}^{\prime}$ & $0 \leq t_{\mathrm{i}}^{\prime} \leq 1, \mathrm{i} \varepsilon\{1, \ldots, \mathrm{N}\}$ \\
Shell permittivities, $\varepsilon_{\mathrm{i}}$ & $1 \leq \varepsilon_{\mathrm{i}} \leq 2, \mathrm{i} \varepsilon\{1, \ldots, \mathrm{N}\}$ \\
Feed HPBW & $30^{\circ} \leq \mathrm{HPBW} \leq 130^{\circ}$ \\
Lens-to-feed distance $h\left[\lambda_{0}\right]$ & $0 \leq h \leq 0.5$ \\
\hline Optimized parameter & Value \\
\hline Normalized thicknesses, $t_{\mathrm{i}}^{\prime}$ & $0.29,0.21,0.17,0.16,0.17$ \\
Shell permittivities, $\varepsilon_{\mathrm{i}}$ & $1.92,1.79,1.62,1.39,1.15$ \\
Feed HPBW & $67^{\circ}$ \\
Lens-to-feed distance $h\left[\lambda_{0}\right]$ & 0.31 \\
\hline
\end{tabular}

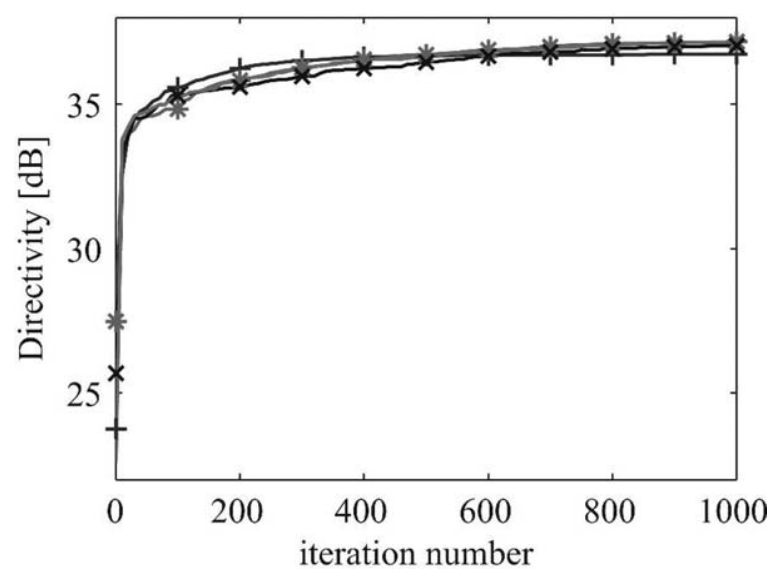

Figure 4 Directivity of a 5 -shell $30 \lambda_{0}$-diameter Luneburg lens fed by a complex source point beam as a function of the PSO iteration number for different number $p$ of particles $[p=10(+), p=20(*), p=30(\times), p=40(-)]$

an all-encompassing approach, where the original version introduced for the used method of optimization (PSO) allows the optimization of not only the lens shell parameters, but also the pertinent feed parameters, like the half power beamwidth (HPBW) and the lens-to-feed distance $(h)$, as represented in Figure 3.

The input and optimized parameters for this problem are reported in Table 1 . The directivity of the Luneburg lens fed by a complex source point beam as a function of the PSO iteration number is reported in Figure 4 for different numbers of particles $p$. The optimum number of particles lies between 10 and 20, not far from the problem dimensionality (12 degrees of freedom). The achieved directivity of the 5-shells optimized lens is equal to $37.2 \mathrm{~dB}$, which compares very favorable with the ideal directivity of a 100 shells Luneburg lens $(38.4 \mathrm{~dB})$.

\subsection{Synthesis of Amplitude Shaped Radiation Patterns}

The goal of this optimization problem is to determine the lens antenna parameters to obtain a radiation pattern, which complies

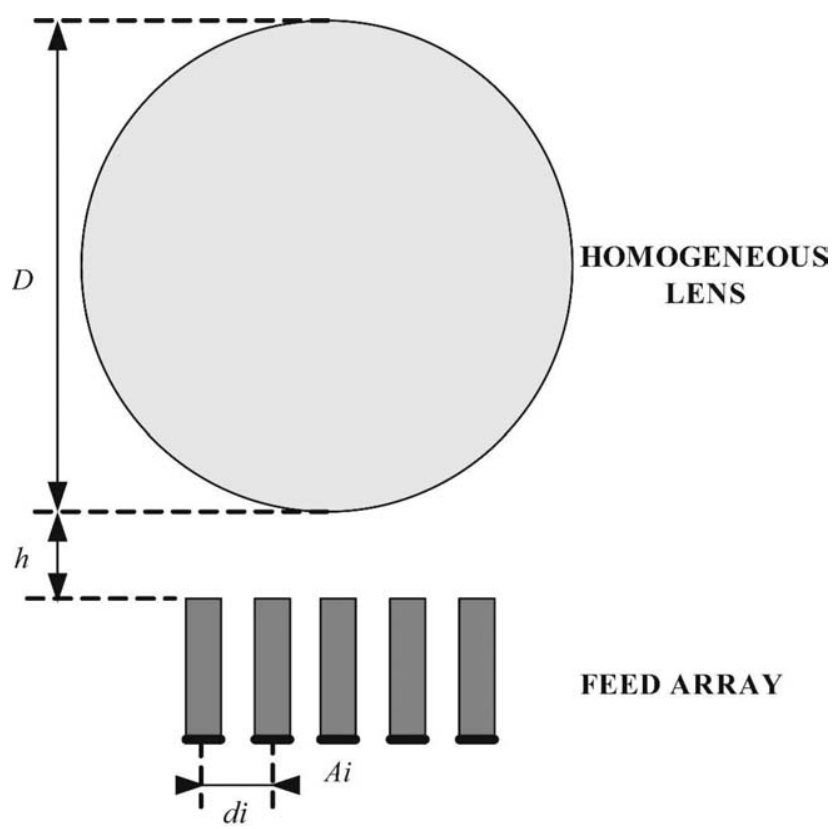

Figure 5 A homogeneous lens of diameter $D$ is illuminated by an array of five open-ended waveguides. The feeds are spaced by a distance $d_{\mathrm{i}}$. The ith waveguide has a weighted excitation denoted $A_{\mathrm{i}}$. The distance between the lens and the array is $h$ 
TABLE 2 Input and Optimized Parameters of the Pattern Shaping Optimization Problem

\begin{tabular}{lc}
\hline Fixed Parameter & Value \\
\hline Lens permittivity & 2.1 \\
Number of feeds & $\mathrm{NF}=5$ \\
\hline Parameter to optimize & Boundaries \\
\hline Lens diameter $D\left[\lambda_{0}\right]$ & $4 \leq D \leq 7$ \\
Lens-to-feed distance $h\left[\lambda_{0}\right]$ & $0 \leq h \leq 1$ \\
Feed amplitude $A_{\mathrm{i}}$ & $0 \leq A_{\mathrm{i}} \leq 1, \mathrm{i} \varepsilon\{1, \ldots, \mathrm{NF}\}$ \\
Feed spacing $d_{\mathrm{i}}\left[\lambda_{0}\right]$ & $0.5 \leq d_{\mathrm{i}} \leq 1.5, \mathrm{i} \varepsilon\{1, \ldots, \mathrm{NF}-1\}$ \\
\hline Optimized parameter & Value \\
\hline Lens diameter $D\left[\lambda_{0}\right]$ & 5.61 \\
Lens-to-feed distance $h\left[\lambda_{0}\right]$ & 0.70 \\
Feed amplitude $A_{\mathrm{i}}$ & $0.65,0.71,0.59,0.72,0.65$ \\
Feed spacing $d_{\mathrm{i}}\left[\lambda_{0}\right]$ & 0.65 \\
\hline
\end{tabular}

with a given amplitude mask (here sectoral). A homogeneous Teflon lens is excited by an array of five open-ended WR 10 waveguides at $75 \mathrm{GHz}$. The geometry of the problem is described in Figure 5, and the input parameters of this optimization procedure are given in Table 2.

The cost function $C$ to minimize is defined as follows:

$$
C=\sum_{\mathrm{i}}\left|f_{\mathrm{MMT}}\left(\theta_{\mathrm{i}}\right)-f_{\text {mask }}\left(\theta_{\mathrm{i}}\right)\right|^{2},
$$

where $f_{\text {mask }}$ is the amplitude mask and $f_{\mathrm{MMT}}$ is the far-field pattern computed by MMT. The sum in Eq. (5) is computed only for the angle positions $\theta_{\mathrm{i}}$ where the radiated field is out of the template.

The far-field patterns are plotted as a function of the number of iterations in Figure 6. The cost function $C$ needs to be evaluated less than one thousand times to reach convergence. The optimized values are given in Table 2. In Figure 7, the optimized far-field pattern computed by MMT is compared with the one simulated with CST microwave studio to validate the accuracy of the analysis method. Although the MMT does not take into account the coupling between the feeds, an excellent agreement is obtained in the upper half space. There are some discrepancies in the lower half space mainly because of the diffraction on the metallic parts of the waveguides.

\subsection{Design of Reconfigurable Lens Antenna}

As a last optimization example, the binary PSO algorithm is used for the design of a reconfigurable dual beam lens fed by an array of 5 open-ended waveguides. By switching ON or OFF

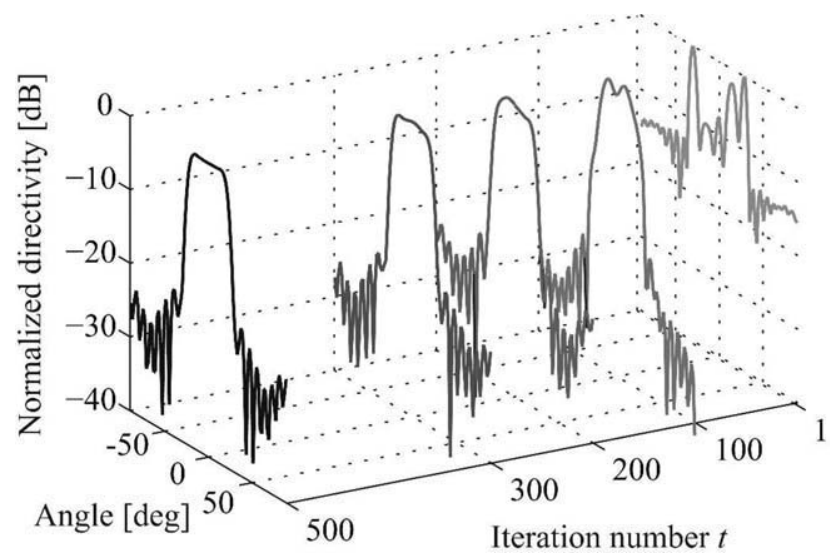

Figure 6 Variation of the far-field patterns, computed at $75 \mathrm{GHz}$ by the MMT, as a function of the number $t$ of iterations

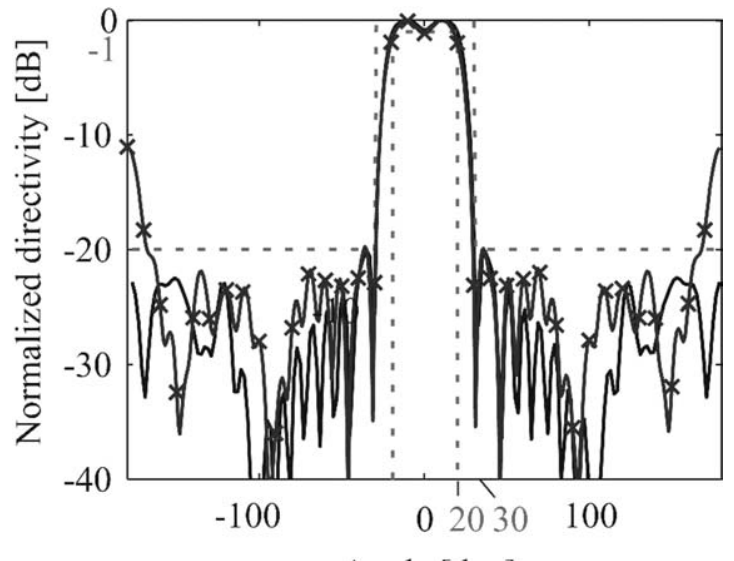

Angle [deg]

Figure 7 Optimized far-field radiation patterns computed by MMT (-) and simulated with CST Microwave Studio (x) with the sectoral amplitude mask (- -)

properly the elements of the feed array, we want the lens antenna to generate either a pencil- or a sector- shaped pattern. For the pencil-shaped pattern, the goal is the maximization of the directivity, whereas the radiation pattern has to fit a given sectoral amplitude mask in the case of a sector-shaped pattern. To measure the optimality of both radiation patterns, one thus adds the cost functions used in examples 3.1 and 3.2. Two lens analysis are therefore required to calculate the global cost function.

The input and optimized parameters of this binary PSO problem are given in Table 3 . The ON or OFF states $A_{\mathrm{i}}$ of the elements of the array are optimized for each configuration whereas the geometry of the lens antenna (lens-to-feed distance $h$ and feed spacing $d_{\mathrm{i}}$ ) remains the same for both configurations. The variables $h$ and $d_{\mathrm{i}}$ are discretized with a $0.05 \lambda$ step. Note also that, the presented optimized results are obtained after $<2000$ iterations.

As shown in Figure 8, if all five waveguides illuminate the lens, a sectoral pattern $\left(+/-30^{\circ}\right.$ angle coverage with a ripple of $<1 \mathrm{~dB}$ and side lobe levels below $-20 \mathrm{~dB}$ ) is achieved. By simply switching off all waveguides except the central one, a $23 \mathrm{~dB}$ pencil beam is obtained. Not taking into account the metallic parts of the waveguides leads here to some discrepancies between the MMT and CST microwave studio. However, these differences are not significant in the region of interest (the upper half space).

TABLE 3 Input and Optimized Parameters of the Reconfigurable Lens Antenna Problem

\begin{tabular}{|c|c|}
\hline Fixed Parameter & Value \\
\hline Lens diameter $D\left[\lambda_{0}\right]$ & 5 \\
\hline Shell permittivities, $\varepsilon_{\mathrm{i}}$ & $1.86,1.57,1.28$ \\
\hline Normalized thicknesses, $t_{\mathrm{i}}^{\prime}$ & $0.57,0.81,1$ \\
\hline Number of feeds & $\mathrm{NF}=5$ \\
\hline Parameter to optimize & Boundaries \\
\hline Lens-to-feed distance $h\left[\lambda_{0}\right]$ & $0 \leq h \leq 1$ \\
\hline Feed spacing $d_{\mathrm{i}}\left[\lambda_{0}\right]$ & $0.5 \leq d_{\mathrm{i}} \leq 1.5, \mathrm{i} \varepsilon\{1, \ldots, \mathrm{NF}-1\}$ \\
\hline Feed amplitude $A_{\mathrm{i}}$ & $A_{\mathrm{i}} \varepsilon\{0,1\}, \mathrm{i} \varepsilon\{1, \ldots, \mathrm{NF}\}$ \\
\hline Optimized Parameter & Value \\
\hline Lens-to-feed distance $h\left[\lambda_{0}\right]$ & 0.15 \\
\hline Feed spacing $d_{\mathrm{i}}\left[\lambda_{0}\right]$ & $1.35,0.50,0.50,1.35$ \\
\hline Feed amplitude $A_{\mathrm{i}}$ (pencil beam) & $0,0,1,0,0$ \\
\hline Feed amplitude $A_{\mathrm{i}}$ (sector beam) & $1,1,1,1,1$ \\
\hline
\end{tabular}




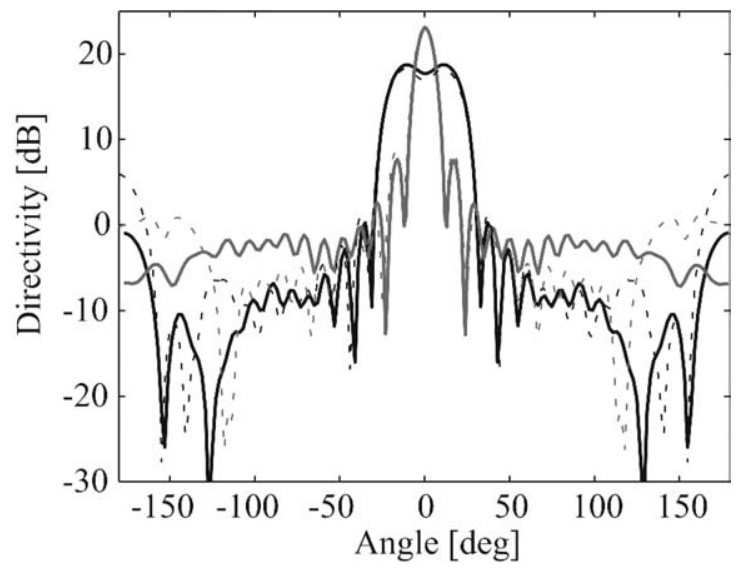

Figure 8 Dual-beam (pencil and sectoral) patterns, obtained by MMT (-) and CST Microwave Studio (- -), achieved by switching-only the waveguides illuminating a 3 -shell lens at $75 \mathrm{GHz}$

\section{CONCLUSION}

A design procedure to optimize spherical lens antennas has been presented. The analysis method is based on a MMT implemented for spherically stratified structures and is combined with a PSO algorithm. Three lens antenna optimization problems are detailed to highlight the potentiality of the proposed methodology. First, both the Luneburg lens parameters and antenna characteristics are optimized to maximize the directivity. Then, the amplitude and position of each element of an array are optimized to shape the beam radiated by a lens antenna to comply with a given farfield mask. Finally, a reconfigurable lens antenna is designed with binary PSO. By switching properly the feeds, a pencil or a sectoral beam can be generated, avoiding thereby the use of any attenuator/amplifier. The good agreement between the optimized far-field radiation patterns computed by MMT and simulated with full wave software validates the proposed methodology.

\section{REFERENCES}

1. B. Schoenlinner, X. Wu, J.P. Ebling, G.V. Eleftheriades, and G.M Rebeiz, Wide-scan spherical-lens antennas for automotive radars, IEEE Trans Microwave Theory Tech 50 (2002), 2166-2175.

2. B. Fuchs, O. Lafond, S. Rondineau, M. Himdi, and L. Le Coq, Offaxis performances of half Maxwell fish-eye lens antennas at 77 GHz, IEEE Trans Antennas Propag 55 (2007), 479-482.

3. J.R. Costa, C.A. Fernandes, G. Godi, R. Sauleau, L. Le Coq, and H. Legay, Compact Ka-band lens antennas for LEO satellites, IEEE Trans Antennas Propag 56 (2008), 1251-1258.

4. C.A. Fernandes, Shaped dielectric lenses for wireless millimeter-wave communications, IEEE Antennas Propag Mag 41 (1999), 141-150.

5. O. Lafond, M. Caillet, B. Fuchs, S. Palud, M. Himdi, S. Rondineau, and L. Le Coq, Millimeter wave reconfigurable antenna based on active printed array and inhomogeneous lens, Eur Microwave Conf, Amsterdam, Holland (2008), 147-150.

6. B. Fuchs, S. Palud, O. Lafond, M. Himdi, and S. Rondineau, Système antennaire dont le diagramme de rayonnement est reconfigurable parmi des diagrammes de rayonnement sectoriels et directifs, et dispositifs émetteur et / ou récepteur correspondant, French Patent 0756664 (Jul. 20, 2007).

7. B. Fuchs, Lentilles stratifiées et sources réelles associées - Analyses théoriques et validations expérimentales en ondes millimétriques, Ph.D. Thesis, University of Rennes 1, France, Nov. 2007.

8. J. Sanford, Scattering by spherically stratified microwave lens antennas, IEEE Trans Antennas Propag 42 (1994), 690-698.

9. S. Rondineau, Modélisation de lentilles sphériques à gradient d'indice et sources conformes associées, Ph.D. Thesis, University of Rennes 1, France, Dec. 2002.
10. B. Fuchs, S. Palud, L. Le Coq, O. Lafond, M. Himdi, and S. Rondineau, Scattering of spherically and hemispherically stratified lenses fed by any real source, IEEE Trans Antennas Propag 56 (2008), $450-460$.

11. L.B. Felsen, Complex-point source solutions of the field equations and their relation to the propagation and scattering of the Gaussian beams, Proc Symp Math 18 (1975), 39-56.

12. J. Kennedy and R. Eberhart, Particle swarm optimization, Proc IEEE Int Conf Neural Netw 4 (1995), 1942-1948.

13. J. Robinson and Y. Rahmat-Samii, Particle swarm optimization in electromagnetics, IEEE Trans Antennas Propag 52 (2004), 397-407.

14. N. Jin and Y. Rahmat-Samii, Advances in particle swarm optimization for antenna designs: Real-number, binary, single-objective and multiobjective implementations, IEEE Trans Antennas Propag 55 (2007), 556-567.

15. J. Kennedy and R. Eberhart, A discrete binary version of the particle swarm algorithm, Proc IEEE Int Conf Syst Man Cybern 5 (1997), 4104-4108.

16. R.K. Luneburg, Mathematical theory of optics, Rhode Island, Providence, Rhode Island, 1944.

17. C.S. Silitonga, Y. Sugano, H. Sakura, M. Ohki, and S. Kozaki, Optimum variation of the Luneberg lens for electromagnetic scattering calculations, Int J Electron 84 (1998), 625-633.

18. B. Fuchs, L. Le Coq, O. Lafond, S. Rondineau, and M. Himdi, Design optimization of multi-shell Luneburg lenses, IEEE Trans Antennas Propag 55 (2007), 283-289.

19. H. Mosallaei and Y. Rahmat-Samii, Non-uniform luneburg and 2shell lens antennas: Radiation characteristics and design optimization, IEEE Trans Antennas Propag 49 (2001), 60-69.

(C) 2010 Wiley Periodicals, Inc.

\section{FILTER DESIGN AND MODE SUPPRESSION INSIDE METAMATERIAL- FILLED RECTANGULAR WAVEGUIDES}

Sibel Çimen, ${ }^{1}$ Gonca Çakir, ${ }^{1}$ and Levent Sevgi ${ }^{2}$

${ }^{1}$ Department of Electronics and Communication Engineering, Kocaeli University, Kocaeli/Turkey; Corresponding author: sibelgunduz@kocaeli.edu.tr

${ }^{2}$ Department of Electronics and Communications Engineering, Doğus University, Zeamet Sok. No.21, Acibadem/Kadiköy, Istanbul, Turkey

\section{Received 13 October 2009}

ABSTRACT: Filter characteristics and mode suppression capabilities inside metamaterial- (MTM) filled rectangular waveguides are investigated. Frequency characteristics of single-negative ( $S N G)$ and double-negative (DNG) finite slength transverse slabs inside rectangular waveguides are numerically simulated. The simulations are performed via in-house-prepared three-dimensional (3D) finite-difference timedomain package; MTM-3D. Simulations are also repeated with the commercial CST Microwave Studio package and results are compared. Tests show that different combinations of SNG and DNG slabs located transversely inside a rectangular waveguide can be used to obtain multi-stopband filters and to suppress modes (i.e., resonances). (C) 2010 Wiley Periodicals, Inc. Microwave Opt Technol Lett 52: 1659-1663, 2010; Published online in Wiley InterScience (www.interscience. wiley.com). DOI 10.1002/mop.25289

Key words: metamaterials; negative permeability; negative permittivity; rectangular waveguides; filter design; mode suppression; finitedifference time-domain method; FDTD

\section{INTRODUCTION}

Rectangular waveguides are widely used as basic guiding structures in many electromagnetic applications. They act as high- 